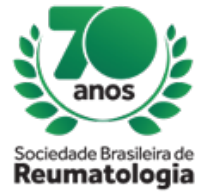

\title{
BEHAVIOR OF CHIKUNGUNYA FEVER IN PSA PATIENTS UNDER BIOLOGICAL TREATMENT
}

Marina Brito (Medicine undergraduate student - Universidade Federal de Pernambuco, Recife, PE, Brasil), Thiago Martins (Medicine undergraduate student - Universidade Federal de Pernambuco, Recife, PE, Brasil), Filipe Mateus Lima (Rheumatology fellow, Clinical Hospital of Pernambuco Federal University, Recife, PE, Brasil), Claudia Marques (Rheumatology, Clinical Hospital of Pernambuco Federal University, Recife, PE, Brasil), Rafaela Gonçalves (Rheumatology, Clinical Hospital of Pernambuco Federal University, Recife, PE, Brasil), Angela Duarte (Rheumatology, Clinical Hospital of Pernambuco Federal University, Recife, PE, Brasil)

\section{BACKGROUND}

Chikungunya fever (CF) is an acute condition, characterized by high fever, rash and disabling musculoskeletal pain. Arthralgia and arthritis are very common, with intense and impeding pain in the daily activities, determining great socioeconomic impact. After acute phase, there may be regression or persistence of the symptoms, with evolution to the chronic phase ( $>3$ months), when joint involvement assumes different clinical patterns. We assessed manifestations of CF in patients with Psoriatic Arthritis (PsA), by a clinical and epidemiological diagnosis, to determine if these patients, due to the use of special classes of drugs that inhibit inflammatory cytokines, reducing local inflammation (immunobiological), would have milder symptoms, or if they would have a more serious condition due to the reduction of immunity by the use of immunobiological and if their previous joint involvement would be a worsening factor for arthralgia.

\section{MATERIALS AND METHODS}

The study was made by the application of a questionnaire by the research team with questions about clinical and epidemiological findings related to $\mathrm{CF}$, the course of their disease and its impact on their basis disease to the PsA patients at a rheumatology department.

\section{RESULTS}

We interviewed 83 psoriatic arthritis patients, and 34 (40.9\%) reported symptoms compatible with CF, whose prevalence was similar with data from the international literature. It was questioned whether people living with patients were affected by $C F$, which was affirmative in $88.2 \%$ of these patients. Worsening was reported in $76.5 \%$ patients after $\mathrm{CF}$, and $23(67.6 \%)$ remained with $\mathrm{CF}$-related pain to date. Complications were reported in 9 (26.5\%) patients with CF, and 5 patients developed PsA after CF, indicating that it may be a possible trigger for development of rheumatological diseases. After CF, treatment was changed in 26 (76.5\%) PsA patients, who were previously using different classes of drugs, indicating change in the course of rheumatologic disease after CF.

\section{CONCLUSION}

It was observed that patients with PsA presented a prevalence of CF similar to general population as described in the literature. Elsewhere in epidemiological analysis we observed high percentage of affected contacts with CF, suggesting that, despite drugs immunosuppressive use, this group of patients does not seem to have an increased risk for arboviruses. Nor was observed the hypothesis that users of the aforementioned medications would present "protective effect" (because the lower intensity inflammatory response) since the incidence of $\mathrm{CF}$ in the studied group was similar to general population. 